\title{
Evidence of errors that have stymied low back pain
}

\begin{abstract}
Background: Treating problems related to the low back can be frustrating because of inadequate justification of long standing; often times with unproven concepts. These beliefs can lead to underwhelming results with this population. Research has revealed inappropriate structural, biomechanical, functional and movement concepts leading to false results and assumptions about low back pain.
\end{abstract}

Purpose: This article will focus on several errors in low back treatment philosophy: the sacrum as a keystone, measurement of movement and mechanics, location of the XYZ axis of the sacroiliac joint (SIJ), intraarticular injections as the gold standard, the disc as the cause of low back pain, testing errors and pelvic asymmetry.

Design/Setting: Specific samples of $\mathrm{x}$-rays, photographs and graphic illustrations were taken from previously published articles by the author.

Methods: The concept of the sacrum functioning as a keystone was analyzed in a functional position. X-rays of the asymmetric pelvis in the long straddle position were compared to X-rays of the symmetrical pelvis in the long straddle position (LSP). Intra-articular injections of the sacroiliac joints were compared to peri-articular injections. Before and after $\mathrm{x}$-rays demonstrated the results of manual correction of a reversible asymmetric pelvis.

Results: With weight-bearing when the ilia are symmetrical there is a bony transverse loading axis at $\mathrm{S} 1$ and movement is on balanced ligaments, negating the concept of function as a keystone. When the ilia are asymmetrical the sacrum was found to flex laterally toward the side of loading and rotate to drive counter rotation of the trunk. Peri-articular injections into the area of the sacroiliac joint are more effective than intra-articular injections. A correction of an asymmetric pelvis to symmetry with a posterior rotation of the innominate on the sacrum was demonstrated. As this movement from asymmetry to symmetry relieves pain so rapidly and completely this lesion is probably more in the nature of a subluxation.

Conclusions: Although the sacrum looks similar to a keystone, it does not
Volume I 3 Issue 6 - 2020

Richard L Don Tigny

Department of Radiology, Northern Montana Hospital, USA

Correspondence: Richard L. DonTigny, Department of Radiology, Northern Montana Hospital PT, 2632 Catron Street \#209, Bozeman, Montana 59718, USA, Tel (406) 219-3416, Email djd@lazy-trout.com

Received: August 27, 2020 | Published: December 30, 2020

function as such, but rather floats on balanced ligaments and is essentially nonweight bearing. A commonly overlooked procedural error has indicated that the sacroiliac joint has minimal motion. X-rays in the long straddle position with counter rotation of the trunk demonstrate about 30 degrees of lateral sacral flexion in each direction. Sacral measurements have been made on $\mathrm{XYZ}$ axes on the sacral plateau even though examination reveals a commonly overlooked, bony, transverse, sacral loading $\mathrm{X}$ axis posterior to sacral S3 at the posterior inferior iliac spines. The gold standard in the use of intra-articular injections for diagnosis of dysfunction of the sacroiliac joint may cause a high percentage $(64 \%)$ of false positive results if injected into the intact capsule. Idiopathic low back pain has no relationship to the herniated disc although a dysfunction of the sacroiliac joint that may cause vertebral instability may be a factor. An anterior rotation of the innominates on the sacrum can cause a reversible pelvic asymmetry. Less than factual assertions and conclusions remain in current consensus. These have precipitated many self-perpetuating myths that have confused investigators and negated carefully considered results in evidence based research.

Keywords: biomechanical errors, etiology of back pain, functional errors, innominate movement, measurement errors, reversible pelvic asymmetry, sacral axes, sacroiliac joint
Abbreviations: LBP, low back pain; LSP, long straddle position; PIIS, posterior inferior iliac spine; PSIS, posterior superior iliac spine; SI, sacroiliac; SIJ, sacroiliac joint; SIJD, sacroiliac joint dysfunction; SL1, primary sacral loading; SL2, secondary sacral loading; PL1, primary pelvic loading; PL2, secondary pelvic loading

\section{Introduction}

Low back pain, idiopathic low back pain, lumbosacral pain, pelvic pain and lumbopelvic pain are non-specific entities often used as diagnoses for pain in the low back. Abraham and Killackey-Jones stated in the Archives of Internal Medicine in 2002 that idiopathic and non-specific low back pain (LBP) have emerged as catchall terms for symptoms that elude physicians' diagnostic ability. They believed that the apparent uncritical acceptance of idiopathic and non-specific LBP as valid concepts has badly affected research and national medical policy. "It is time to re-think this position." The validity of non-specific or idiopathic LBP seems to be a historical myth."

This paper draws from over 50years of research into the mechanics of the pelvis and of idiopathic low back pain. In 1964 when I saw a patient with low back pain and the next day she was free of pain as a result of falling from a tractor, I realized that she had a reversible, biomechanical lesion and if I could understand the nature of this lesion, I could correct it in the clinic... without the use of a tractor. I fabricated biomechanical models and developed a simple method of treatment. What follows are some research based reflections on the 'mechanics of the pelvis and of common pain in the low back in relation to errors that are pervasive but questionable in the treatment of low back and sacroiliac (SI) pain.

\section{The keystone concept}

Vleeming has proposed that the pelvis is made stable, when weight bearing, by a self-locking mechanism of form and force closure based on the sacrum as the keystone of an arch. ${ }^{2}$ A keystone is a wedge at the top of an arch that holds the arch tightly together with weight loading. Vleeming's biomechanical model is in error because the sacrum is actually suspended from the ilia by the dense posterior interosseous ligaments and functions as the reverse of a keystone by hanging more deeply between the ilia with increased weight loading. ${ }^{3,4}$ The posterior superior iliac spines (PSIS) approximate and block further movement of the sacrum downward between the innominates.

Vukicevic and his colleagues found that as long as the posterior 
interosseous ligaments are intact the joints do not approximate with weight-loading. ${ }^{5}$ If the sacrum functioned as a keystone, the joint surfaces would approximate and the PSIS would diverge. The superincumbent weight is transferred from the sacrum through the posterior interosseous ligaments to the innominate bones and not through the sacroiliac joints (SIJ). ${ }^{7-9}$ Movement is on a transverse, bony, sacral loading axis ${ }^{6-8}$ and keystones have no loading axis. It is critical to note that this axis is at the SI joints at the most narrow aspect of the sacrum and located so that with loading both of these joints tend to fall away from the innominates restricted only by ligaments.

Primary sacral loading of the superincumbent weight (SL1) on the posterior interosseous ligaments is equal to the secondary sacral loading (SL2) on the sacrotuberous and sacrospinous ligaments (SL1=SL2) (Figure 1). Primary pelvic loading (PL1) is equal to the secondary pelvic loading (PL2) thus PL1=PL2. The balanced loading on these forces creates multiple interchangeable force couples Force couples function to modify, absorb and redirect forces such as linear and angular acceleration and deceleration, linear and angular momentum, impact loading and unloading and others. Force couples help to enhance function, preserve the systems, and prevent injury. Form and force closure and self-bracing probably occur in the nonweight bearing pelvis or in the cadaver pelvis, but once the sacrum is loaded with the superincumbent weight everything changes. The concept of a keystone cannot be applied to describe this loading.

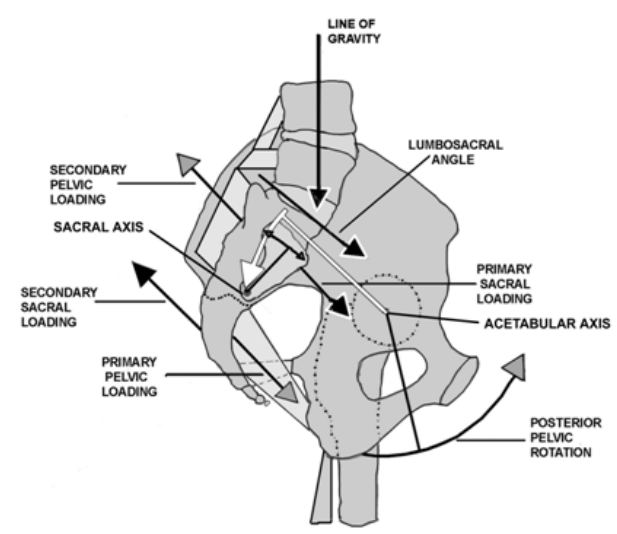

Figure I Primary sacral loading (SLI) occurs on the posterior interosseous ligaments with an instantaneous equal secondary loading (SL2) of the sacrotuberous ligaments in the opposite direction. The lumbar lordosis is increased with the primary sacral loading, as is the lumbosacral angle. ${ }^{7,8}$ Because the line of gravity is posterior to the acetabular axis, loading of the sacrum causes a simultaneous sequential loading of the pelvis (PLI) resulting in a posterior pelvic rotation on the acetabular axis. The secondary loading of the pelvis (PL2) further balances the ligaments, decreases the lordosis and decreases the lumbosacral angle. ${ }^{7-9}$

\section{Measurement of movement and mechanics}

Sturesson in $1989^{10}$ used tiny tantalum balls implanted in the pelvis, measured pelvic movement in the long straddle position (LSP) and reported finding about 2 degrees of movement at the SIJ. Smidt in $1995,{ }^{11}$ using a different method for the measurement of movement of the SIJ in the LSP, reported finding about 30 degrees of motion.

There were no obvious errors in measurement, but Sturesson had blocked the pelvis in the frontal plane in the LSP for his measurements. ${ }^{10}$ In the LSP the pelvis is asymmetric and oblique to the line of travel to extend the length of the stride. When Sturesson blocked the pelvis he inadvertently blocked it in a position of symmetry and simply measured the symmetrical pelvis in the LSP with the right leg advanced and again with the left leg advanced. ${ }^{10}$ All he measured was a minimal movement in the symmetrical pelvis. This was a fatal error in procedure.

I had myself $\mathrm{x}$-rayed in the LSP with the pelvis in asymmetry with counter rotation right and left (Figures 2\&3). ${ }^{8,9}$ The x-rays clearly showed the posterior rotation of the innominate bone on the side of loading moving the sacrum caudad, but not moving caudad on the sacrum. The innominate on the contralateral side lifts the sacrum on that side to cause lateral sacral flexion with rotation toward the side of loading.
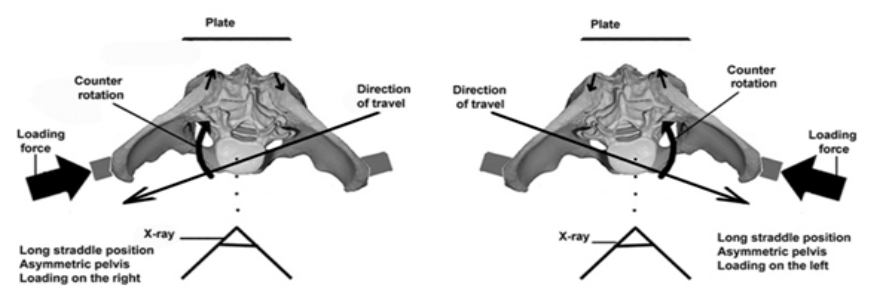

Figure 2 Procedure to demonstrate pelvic movement. In order to view innominate movement on the oblique asymmetric pelvis it was necessary to have the front of the pelvis square to the camera, but oblique to the line of travel.

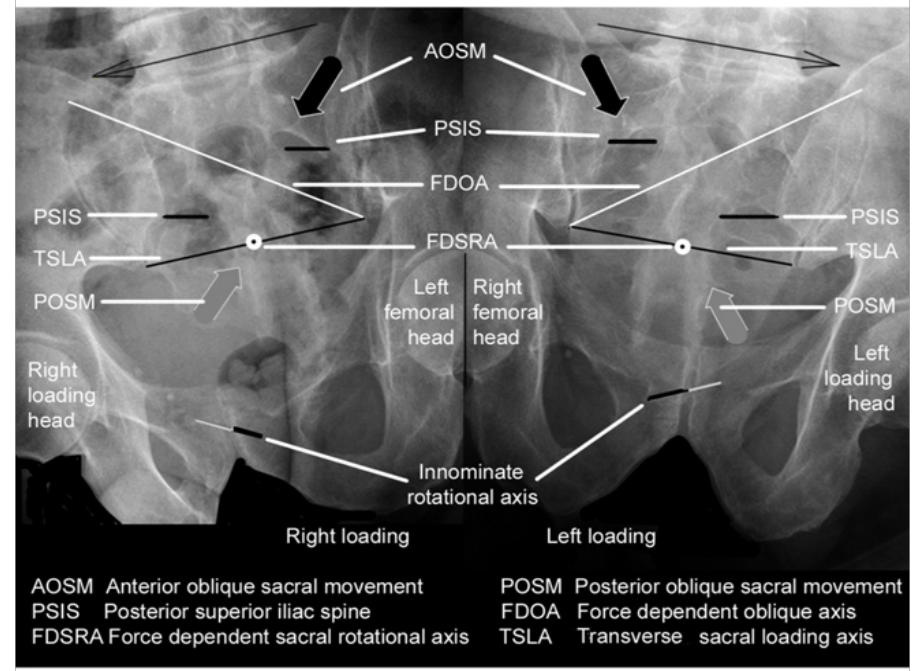

Figure $3 \mathrm{X}$-rays taken of a pelvis in the long straddle position with counter rotation and with loading to the right and to the left. Note the location of the transverse sacral loading axis. 
I also did one view in the LSP with the pelvis blocked in the frontal plane, as per Sturesson and the symmetrical pelvis in that position is obvious (Figures $4 \& 5)^{7-9}$

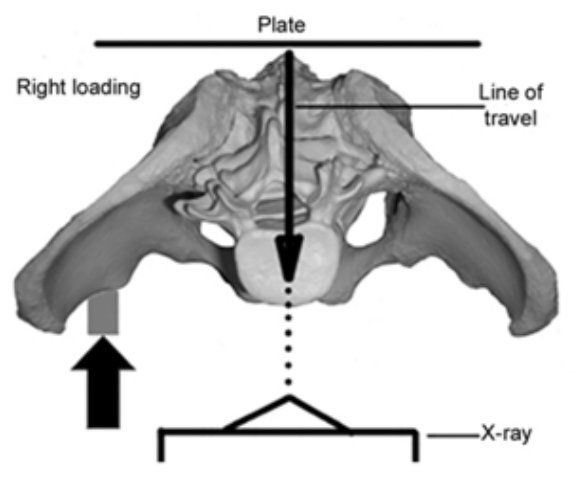

Figure 4 Procedure as per Sturesson. Pelvis is frontal to the $x$-ray and the line of travel is directly toward the $\mathrm{X}$-ray.

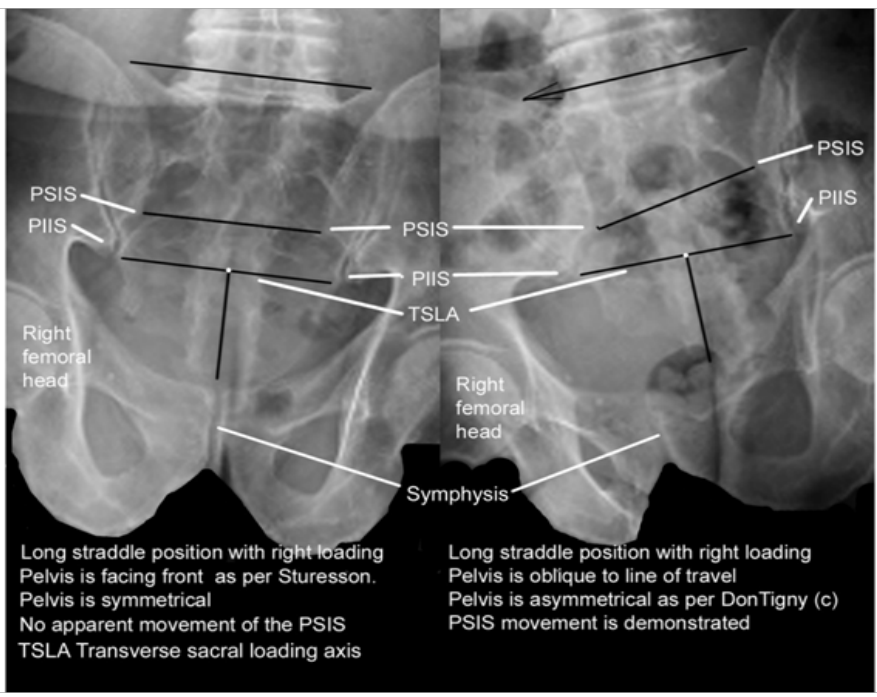

Figure 5 Comparison of $\mathrm{x}$-rays.

In the x-ray on the left the lack of movement at the PSIS demonstrates that the pelvis is symmetrical even though the subject is in the long straddle position. Note in the right $\mathrm{x}$-ray that with posterior rotation on the loaded side the innominate causes the sacrum to move caudad, but does not move caudad on the sacrum.

With a dysfunction of the SIJ in anterior rotation, the innominates sublux cephalad and laterally on the sacrum, which limits the normal motion and function of the SIJ. The transverse sacral axis is disrupted, but can still have some limited function moving on a pathological axis. ${ }^{6-9,14}$ Sturesson ${ }^{12}$ made his measurements on patients with unilateral and bilateral dysfunction of the SIJ, which would have further decreased the excursion of the normal SIJ.

Not only did Sturesson err in his SIJ movement assessment, but also in where the coordinates should have been located. In the LSP when the innominates move the sacrum into lateral flexion a force-dependent oblique axis is created and the sacrum then moves on that oblique axis to drive counter rotation of the spine..$^{6-9,14}$

Attempts to replicate Sturesson's work may make the same fatal error in procedure. Anyone who uses Sturesson's work as a reference of evidence of non-movement of the SIJ may be in error. In reality the immobile SIJ is now a self-perpetuating myth, ${ }^{1}$ which has effectively blocked both evidenced-based research for the cause of low back pain and serious study of the SIJ. The use of tiny tantalum balls is an excellent method of measurement, but the x-ray must be frontal to the oblique pelvis in the LSP and not with the pelvis frontal to the line of travel as shown in the previous figures.

\section{Location of the $X Y Z$ axes of the sacroiliac joint}

Sturesson calculated that 2000 patients had been investigated by 1990 with implanted tantalum balls and using the XYZ coordinates for the sacroiliac joints on the center of the sacral plateau. ${ }^{12}$ In writing for Vleeming's book (6) I noticed that the ilial tuberosities would not allow a transverse axis for the sacroiliac joint at S2, but forced the axis caudad to the posterior inferior iliac spines (PIIS) at S3 where it functions as a bony, transverse, sacral $\mathrm{X}$ axis (Figure 6). This was verified by Gracovetski, ${ }^{13}$ who found a bony transition point at the PIIS, The transverse sacral loading axis alters the traditional location of the XYZ coordinates from the sacral plateau.

Not only did Sturesson err in his SIJ movement assessment, but also in where the coordinates should have been located. In the LSP when the innominates move the sacrum into lateral flexion a force-dependent oblique axis is created and the sacrum then moves on that oblique axis to drive counter rotation of the spine (Figure 6). ${ }^{6-9,14}$

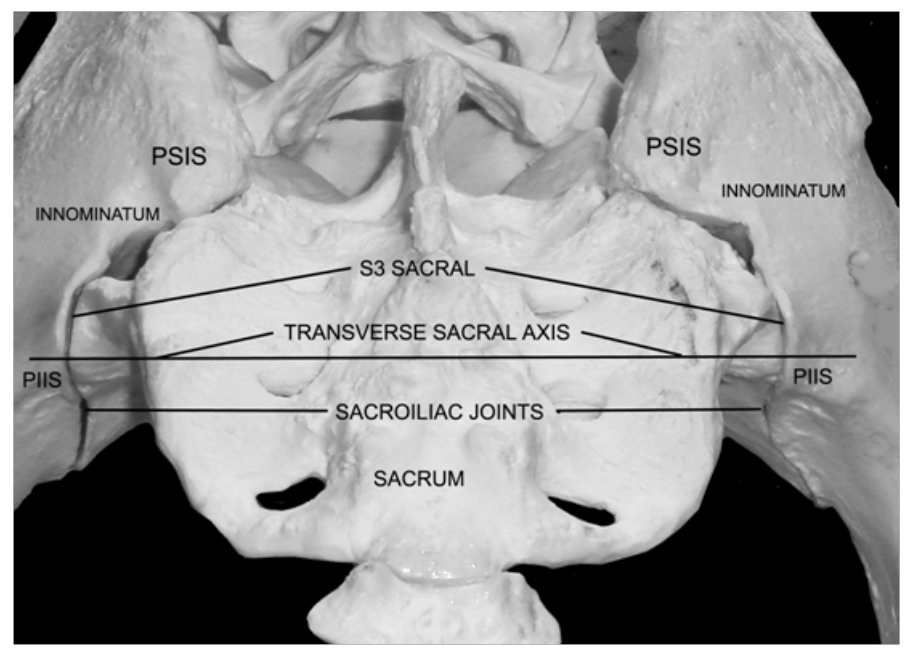

Figure 6 The transverse sacral loading axis (The $X$ axis).

Note how the iliac tuberosities overlie the sacrum and prevent any movement in posterior rotation of the innominates on the sacrum. Take particular note of the location of the posterior inferior iliac spines.

With a dysfunction of the SIJ in anterior rotation, the innominates sublux cephalad and laterally on the sacrum, which limits the normal motion and function of the SIJ. The transverse sacral axis is disrupted, but can still have some limited function moving on a pathological axis. 
${ }^{6-9,14}$ Sturesson ${ }^{12}$ made his measurements on patients with unilateral and bilateral dysfunction of the SIJ, which would have further decreased the excursion of the normal SIJ.

\section{Intra articular injection into the sacroiliac joint as a gold standard}

Murikami and his colleagues found the gold standard for sacroiliac dysfunction in the use of intra articular injections for diagnosis of dysfunction of the SIJ may cause a high percentage (64\%) of false positive results if injected into the intact capsule, which encapsulates the injection. ${ }^{5}$ They compared periarticular and intra articular injections of the sacroiliac joint for the relief of low back pain. Using periarticular injections in 25 consecutive patients with low back pain they found that it was effective in all patients. In a comparable group, intra articular injections were effective in 9 of 25 patients. An additional 16 patients who had no relief from the initial intra articular injection were all relieved with a periarticular injection. ${ }^{15}$

The improvement rate after periarticular injection was 96\% compared to $36 \%$ for the intra articular injection. They concluded that for SIJ pain periarticular injection is more effective and easier to perform than the intraarticular injection and should be tried initially. ${ }^{15}$

\section{The disc as the cause of low back pain}

Another commonly accepted and often repeated myth is that degeneration of the intervertebral disc is a major source of low back pain. Holt ${ }^{16,17}$ who also found that degeneration of the disc is common and commonly found in subjects with no history of back pain, concluded that pain production is not necessarily an indication of an abnormal disc. Savage stated that $47 \%$ of those who had experienced low back pain had normal lumbar spines. ${ }^{18}$

Nachemson ${ }^{19}$ observed that "Even though in elective, carefully selected lumbar disc surgery, $90 \%$ of the patients will enjoy the relief of leg pain, as many as $60-70 \%$ may continue to have some low back pain. If a relationship existed between the disc and low back pain, then the low back pain should be relieved with the repair of the disc.

The frequency and level of degeneration is of greater interest as the prevalence of disc degeneration at L4/5 and L5/S1 reported was three times greater than that at L3/L4 and above. ${ }^{20}$ With a dysfunction of the SIJ in anterior rotation the lumbosacral angle will be increased and the iliolumbar ligaments will be loosened, destabilizing the lower lumbar vertebra and increasing shear and torsion shear to the disks. Also with this dysfunction, the innominates may sublux cephalad and laterally on the sacral S3 segment causing a vertical shear on the dual origin of the piriformis and may cause piriformis syndrome. ${ }^{6-9,14}$ This movement may also cause the sacral origin of the gluteus maximus to separate from its ilial origin on a line from the PSIS to the trochanter and may cause pain down the iliotibial band and into the lateral capsule of the knee.

In 1992, Shaw reported on 1000 consecutive cases of low back pain using changes in apparent leg length and movement of the pelvis from asymmetry to symmetry to correctly identify and treat the dysfunction of the SIJ. ${ }^{21} \mathrm{He}$ found that $98 \%$ of all patients had at least some degree of dysfunction of the sacroiliac joint (SIJD) and his surgical incidence for herniated discs dropped to $0.2 \% .{ }^{21}$ The improvement of 50 consecutive cases of SIJD of $96 \%$ in Murikami's study ${ }^{14}$ is nearly equal to an incidence of $98 \%$ as shown in Shaw's study.

\section{Testing errors}

White reported at The American Academy of Orthopedic Surgeons that they were able to arrive at a firm diagnosis for low back pain only about $15 \%$ of the time. ${ }^{22}$ Conventional tests for low back pain generally have an unsatisfactory inter-rater reliability of only about $25-30 \%{ }^{23,24}$ and are non-specific, inappropriate and not helpful because they were developed without serious consideration of the sacroiliac joints as a major source of back and pelvic pain.

\section{Up-slips, posterior dysfunctions and reversible pelvic asymmetry}

James Mennell ${ }^{25}$ described both anterior and posterior dysfunctions of the SIJ in detail. Up-slips and posterior dysfunctions of the SIJ are traditionally diagnosed through the identification of local pain in the SIJ that is increased with passive straight leg raising (PSLR), a short leg on the more painful side and an asymmetric pelvis (Figure 7). This is commonly treated in the traditional side-lying method of manipulation, pulling the shoulder back and shoving the hip anteriorly and caudad hard enough to cause cavitation. This method of correction is potentially harmful, may be a source of chronic joint dysfunction and may even be contra indicated. Studies have found that a primary dysfunction in posterior rotation is not possible because of the structural and functional position of the iliac tuberosities. The SIJ must be treated as a primary dysfunction in anterior innominate rotation..$^{6-9,26}$

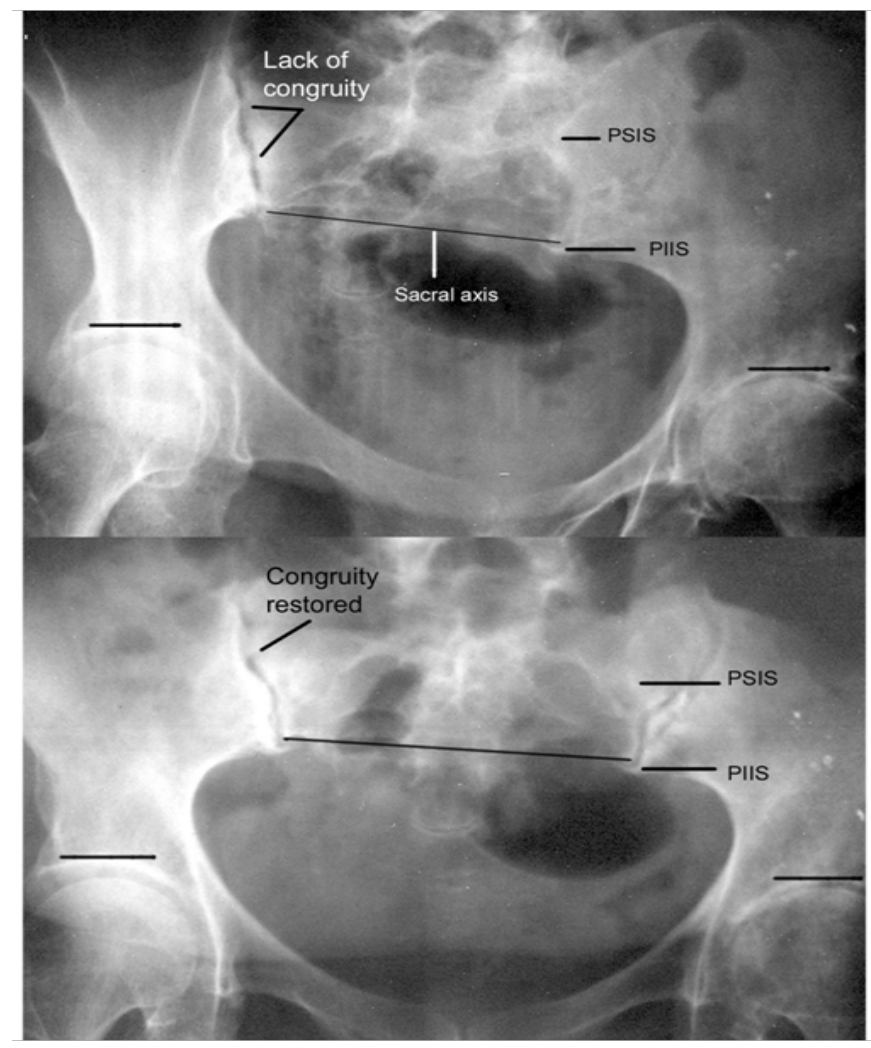

Figure $7 \mathbf{A}$ reversible pelvis asymmetry.

Upper: An asymmetric pelvis with a short right leg, Lower: Symmetry is restored with a bilateral posterior rotation of the innominates on the sacrum. Note how the sacral axis is properly seated on both sides. The patient was free of pain following correction of the innominates posteriorly on the sacrum to the balanced position. 
The dysfunction in anterior rotation loosens the iliolumbar ligaments, the sacrotuberous ligaments, destabilizes the lumbosacral vertebra and increases the lumbosacral angle increasing shear and torsion shear to the disk. It may also be a factor in spondylolisthesis. ${ }^{6-9}$ Practitioners, who use the traditional side-lying method of correction, are at risk of increasing shear at L4,5-S1, joint instability, ligamentous laxity and disc herniation. ${ }^{6-9}$

\section{Discussion}

The sacrum hangs suspended from the symmetrical innominates and is essentially non-weight bearing: the superincumbent weight being transferred through the posterior ligaments. ${ }^{8}$ During normal gait, when the pelvis moves into asymmetry, the innominate rotation causes the sacrum to rotate on the mid-sacral loading axis, flexes laterally toward the side of loading and creates a force-dependent, oblique axis. ${ }^{6-9,27-29}$ Lateral sacral flexion is an unstable position. A controlled instability ${ }^{13}$ causes the sacrum to move on that oblique axis to drive counter rotation of the spine and decrease loading forces to the femoral head. The piriformis and the sacral origin of the gluteus maximus act as prime movers for the sacrum in restoring it to the position of symmetry at mid-stance. ${ }^{6-9,28}$ This has major, commonly overlooked, biomechanical effects on normal gait. ${ }^{7,27}$ Lateral sacral flexion is easily palpable when creating an asymmetric pelvis in the sitting position..$^{6-8}$

\section{Conclusions}

The keystone concept immobilized the pelvis in the minds of most researchers and this immobility was solidified by the lack of pelvic motion as measured by Sturesson. These critical errors have coerced researchers to seek other mechanisms as causative factors. Professionals are using non-specific testing to identify vague neurological hypotheses and inadequately proven concepts, such as classification, lumbar stenosis and the disk in attempts to identify low back pain and may be missing successful, more research supported options for their patients. There are whole areas of low back pain and pelvic pain evaluation and treatment that are left virtually unexamined because they conflict with current orthodoxy.

Idiopathic low back pain is a commonly overlooked, reversible, biomechanical, dysfunction of the sacral axis in anterior innominate rotation, usually bilateral and asymmetrical. ${ }^{6-9,23,27-30}$ Frequently caused by a shift in the line of gravity anterior to the acetabula, when lifting, bending, lowering, shoveling, sweeping, etc., the anterior innominate rotation loosens the sacrotuberous ligaments disturbing the ligamentous balance. The innominates sublux on an acetabular axis; shear cephalad and laterally on the sacrum at S3 and fixate in that position. This puts a vertical shear on the dual origin of the piriformis muscle at the PIIS and may cause a separation of the sacral origin of the gluteus maximus from its ilial origin at the PSIS. Diagnosis of SIJD is simply by identifying the painful point at the PIIS and noting how the leg appears to shorten with elevated traction. All of these symptoms are reversed with a correction in posterior rotation of the innominates on the sacrum..$^{6-9}$

\section{Acknowledgements}

The author is grateful to Eric Wilting, John Rosenbaum and the Radiology department of Northern Montana Hospital, Havre, Montana, for their expert assistance.

\section{Conflicts of interest}

Author declares that there are no conflicts of interest.

\section{Funding}

None.

\section{References}

1. Abraham I, Killackey-Jones B. Lack of evidence-based research for idiopathic low back pain. Arch Intern Med. 2002;162(13):1442-1444.

2. Vleeming A. The Sacroiliac Joint. A clinical-anatomical, biomechanical and radiological study. Netherlands: Erasmus University; 1990.

3. Grant JCB. A Method of Anatomy: Descriptive and Deductive. $6^{\text {th }}$ edn. Baltimore, USA: Williams \& Wilkins; 1958.

4. Weisl H. The Relation of Movement to Structure in the Sacroiliac Joint. Doctoral Thesis. Manchester, England: University of Manchester; 1953

5. Vukicevic S, Marusic A, Stavljenic A. Holographic analysis of the human pelvis. Spine. 1991;16(2):209-214.

6. DonTigny RL. A detailed and critical biomechanical analysis of the sacroiliac joints and relevant kinesiology: the implications for lumbopelvic function and dysfunction. In: Vleeming A, Mooney V, Stoeckart R, editors. Movement, Stability \& Lumbopelvic Pain: Integration of research and therapy. Edinburgh, USA; Churchill Livingstone (Elsevier); 2007. p. 265-279

7. http://thelowback.com/pdfs/toc-pro.pdf

8. DonTigny RL. Sacroiliac 101: Form and Function - A Biomechanical Study. J of Prolotherapy. 2011;3(1):561-567.

9. Don Tigny RL. Sacroiliac 201: Dysfunction and Management A biomechanical solution. $J$ of Prolotherapy. 2011;3(2):644-652.

10. Sturesson B, Selvik G, Uden A. Movements of the sacroiliac joints. A roentgen stereophotogrametric analysis. Spine. 1989;14:162-165.

11. Smidt GS, McQuade K, Wei SH, et al. Sacroiliac kinematics for reciprocal stride positions. Spine. 1995;20(9):1047-1054.

12. Sturesson B. Movement of the sacroiliac joint with special reference to the effect of load. In: Vleeming A, Mooney V, Stoeckart R, editors. Movement, Stability \& Lumbopelvic Pain: Integration of research and therapy. Edinburgh, USA: Churchill Livingstone (Elsevier); 2007. P. 345

13. Gracovetsky S. Stability or controlled instability. In: Vleeming A, Mooney V, Stoeckart R, editors. Movement, Stability \& Lumbopelvic Pain: Integration of research and therapy. Edinburgh, USA: Churchill Livingstone (Elsevier); 2007. p. 279-293

14. Don Tigny RL. Anterior dysfunction of the sacroiliac joint as a major factor in the etiology of idiopathic low back pain syndrome. Phys Ther. 1990;70(4):250-265.

15. Murakami E, Tanaka Y, Aizawa T, et al.Effect of periarticular and intraarticular lidocaine injections for sacroiliac joint pain: Prospective comparative study. J of Orthopaedic Science. 2007;12(3):274-280.

16. Holt EP. Fallacy of cervical discography: Report of 50 cases in normal subjects. JAMA. 1964;188(9):799-801.

17. Holt EP. Fallacy of lumbar discography. J Bone Joint Surg (AM). 1968;50:720-726.

18. Savage RA, Whitehouse GH, Roberts N. The relationship of the magnetic resonance imaging appearance of the lumbar spine \& low back pain, age and occupation in males. Eur Spine J. 1997;6(2):106-114. 
19. Nachemson A, Morris JM. In vivo measurements of intradiscal pressure. J Bone Joint Surg Am. 1964;46:1077-1092.

20. Pool-Goudzwaard AL, Hoek van Dijke G, Vleeming A et al. The iliolumbar ligament influence on the coupling of the sacroiliac joint and the L5-S1 segment. In: Vleeming A, et al. editors. The Third Interdisciplinary World Congress on Low Back and Pelvic Pain. 19-21 November, Vienna, Austria; 1998. p. 313-315

21. Shaw JL. The role of the sacroiliac joint as a cause of low back pain and dysfunction. In: Vleeming A, et al. editors. The First Interdisciplinary World Congress on Low Back Pain and its Relation to the Sacroiliac Joint. San Diego, USA; 1992. p. 67-80

22. White AA. Introduction. In: White AA, Gordon SL, editors. American Academy of Orthopaedic Surgeons Symposium on Idiopathic Low Back Pain. St. Louis, USA: CV Mosby Co; 1982. p. 223

23. Flynn T, Fritz J, Whitman J, et al. A clinical prediction rule for classifying patients with low back pain who demonstrate short-term improvement with spinal manipulation. Spine. 2002;27(24):2835-2843.
24. Fritz, JM, Cleland JA, Childs JD. Sub grouping Patients with Low Back Pain: Evolution of a Classification Approach to Physical Therapy. $J$ Orthop Sports Phys Ther. 2007;37(6):290-302.

25. Mennell JB. The Science and Art of Joint Manipulation. Postgrad Med J. 1940;16(172):67.

26. Tingren J, Soinila S. Reversible pelvic asymmetry. J of Manipulative and Physiological Therapeutics. 2006;29(7):561-565.

27. Don Tigny RL. Critical analysis of the functional dynamics of the sacroiliac joints as they pertain to normal gait. $J$ of Orthopaedic Medicine (UK). 2005;27:3-10.

28. Don Tigny RL. Dysfunction of the sacroiliac joint and its treatment. JOSPT. 1979;1:13-25.

29. Don Tigny RL. Function and pathomechanics of the sacroiliac joint Phys Ther. 1985;65(1):35-44.

30. Davis P, Lentle BC. Evidence for sacroiliac disease as a common cause of low backache in women. Lancet. 1978;2(8088):496-497. 